This is not a peer-reviewed paper.

Pp. 206-209 in Soil Erosion Research for the $21^{\text {st }}$ Century, Proc. Int. Symp. (3-5 January 2001, Honolulu, HI, USA).

Eds. J.C. Ascough II and D.C. Flanagan. St. Joseph, MI: ASAE.701P0007

\title{
A Laser Distance-Based Method for Measuring Standing Residue ${ }^{1}$
}

\author{
F. A. Fox and L. E. Wagner ${ }^{2}$
}

\begin{abstract}
Crop residue standing above the soil surface is 5 to 10 times more effective in preventing wind erosion than the same mass of residue lying flat on the soil surface. Managing tillage operations and designing tillage tools to preserve standing residue require accurate and objective measurement before and after field operations. Manual methods of counting and measuring standing residue provide accurate but slow measurements, making it costly to obtain representative samples for field-size areas. A device was developed using a laser distance sensor to obtain estimates of stem counts, width, and height. These estimates were compared to values obtained using manual counting and measurement. At a scan speed of 2 meters per minute in wheat straw with a stem population of 800 per square meter, counts were estimated accurately but width and height were underestimated consistently.
\end{abstract}

Keywords. Wind erosion, Standing residue, Air quality, Laser scanning.

\section{Background}

The erosion of soil by wind, i.e., wind erosion, is a problem in arid and semi-arid regions around the world. During an erosion event, the wind carries away the most productive fraction of the soil, thus threatening the longterm sustainability of agriculture in affected regions. Poor visibility from dust storms also can result in automotive chain collision accidents and loss of life. The fine particulate components, PM-10 and PM-2.5, in airborne dust are respiratory health hazards for people and animals in downwind areas. Finally, wind erosion deposition contributes to water pollution and increases infrastructure maintenance costs for both the public and private sectors.

The management of surface residues is a critical component of successful strategies for wind erosion control. Residue that remains standing above the soil surface is much more effective than equivalent amounts of residue lying flat on the soil surface in reducing or eliminating the potential for wind erosion (Hagen, 1996). At present, the only method available for nondestructively measuring standing residue involves manually counting and measuring individual stems. This method is accurate for the small areas evaluated but is time and labor intensive. Thus an accurate assessment of standing residue on field size areas requires a large investment of time and effort. Farmers and Natural Resource and Conservation Service personnel need a tool that will quickly and objectively estimate standing residue on land susceptible to wind erosion. Such a device would allow tillage equipment manufacturers to readily assess the performance of machines designed to preserve standing residue. Researchers also could evaluate the effects of field operations and natural processes on standing residue, allowing better development, verification, and extension of models. Measuring actual field conditions is fundamental to developing and implementing processbased analysis tools, conservation strategies, and management plans.

The main effect of standing residue that prevents wind erosion is to reduce the velocity of the wind near the soil surface. Hagen and Armbrust (1994) developed relationships between the silhouette area index and both the aerodynamic roughness length and the reduction in wind shear stress at the soil surface. The silhouette area index, sai, is defined as:

${ }^{1}$ Contribution from the USDA-ARS in cooperation with the Dept. of Agronomy, Dept. of Biological and Agricultural Engineering and the Kansas Agricultural Experiment Stattion, Contribution No. 01-69-A.

${ }^{2}$ Fred. A. Fox Jr., Agricultural Engineer, Wind Erosion Research Unit, USDA-Agricultural Research Service, 1007 Throckmorton, Kansas State University, Manhattan, KS, 66506; Larry. E. Wagner, Wind Erosion Research Unit, USDA-Agricultural Research Service, 1007 Throckmorton, Kansas State University, Manhattan, KS, 66506. Corresponding Author: Fred. A. Fox Jr., Wind Erosion Research Unit, USDA-Agricultural Research Service, 1007 Throckmorton, Kansas State University, Manhattan, KS, 66506; tel: (785) 532-6694; fax: (785) 532-6528; <fredfox@weru.ksu.edu>. 


$$
s a i=\frac{1}{A} \sum_{i=1}^{n} d_{i} h_{i}
$$

where $i$ is the index, $n$ is the total number of stalks, $h_{i}$ is the height of an individual stalk, $d_{i}$ is the diameter of the stalk and $A$ is the ground area over which the stalks are distributed. This shows that the measurement of interest in wind erosion research is the area of the plant that is presented to the wind or a side view of the plant elements. Musick et al (1996) also showed in wind tunnel testing that the areal distribution of plant stalks is equally important in determining the distribution of the wind shear force on the soil surface. Therefore, a measuring device for standing residue should be able to quantify the height, the number of standing stalks, and the distribution over the surface.

Nondestructive methods for measuring standing residue can be divided into groups based on the type of detection and the amount of information returned. Devices that detect the presence of standing residue within a set distance from the sensor return a distribution of standing residue along a single axis, such as the number of stems along a row. Methods falling into this category are a mechanical whisker, a reflectance sensor, and a light source and sensor pair. Height distribution can be obtained by stacking multiple devices.

A device that views the standing residue over an area, such as image acquisition and analysis, obtains both residue height and distribution in one direction. This type of method normally requires some way of defining a cutoff, because continuous standing stubble eventually will completely block the line of sight. Top-down views of standing residue also could return a distribution of standing residue over an area, but then would not return any height information.

Lastly, a device that obtains a distance when pointed at an object can return both a distribution of standing residue over the surface of the ground and, if partly angled toward the ground, partial height information. A laser distance sensor provides high resolution distance sensing. Sonar distance sensors are limited to measuring the distance to objects much larger than the individual stalks of interest in this application.

The objective of this study was to determine if the laser distance method of measuring standing residue can accurately and repeatably measure the silhouette area index for a small grain stubble.

\section{Methods}

The laser distance sensor used in this study, a Nova Ranger NR$40,{ }^{3}$ uses the principle of triangulation to determine the distance from the sensor to a 4.8 millimeter diameter spot that the laser light projects onto an object. The laser distance measurement is made at an angle to the horizontal, as illustrated in Figure 1, and two distances can be extracted: $l_{s}$, the horizontal distance from the sensor to the stem and $h_{s}$, the vertical distance from the sensor datum to the point where the laser spot illuminates the stem. This angled geometry also places the line of sight above the stems, reducing the frequency of blocked views of the laser spot. The estimate of the stem height distribution determined will be a minimum envelope, indicating only that the stems were at least this tall, since maximum stem height is not measured. As for a horizontal distance measurement, a better estimate of the stem height distribution can be obtained only with multiple scans of the same region.

Referring to Figure $1, l_{s}$, the horizontal distance from the sensor to the laser light spot on the stem is:

$$
l_{s}=d \sin \theta
$$

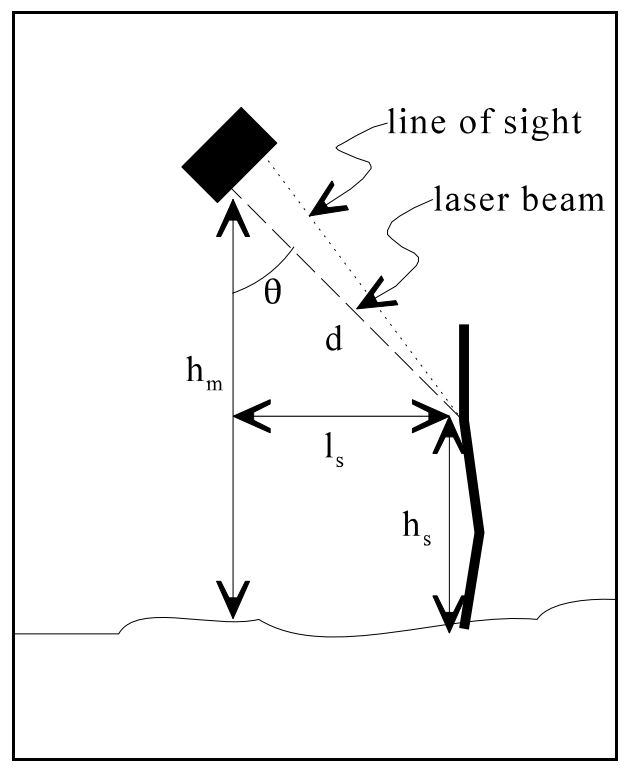

Figure 1. Stem distance geometry.

where $d$ is the laser distance measurement, and $\theta$ is the angle between the laser beam and the vertical axis. Similarly, $h_{s}$, the vertical distance from the sensor datum to the point where the laser spot illuminates the stem is:

\footnotetext{
${ }^{3}$ Model and manufacturer provided for information only and does not imply USDA endorsement.
} 


$$
h_{s}=h_{m}-d \cos \theta
$$

where $h_{m}$ is the height of the sensor above the sensor datum. For determining residue height, it is important that the sensor datum represent the location of the ground surface. If the angle $\theta$ is accurately known and the residue is not too dense, measurements taken will include distances to the ground surface, allowing the sensor datum to be established from the measurements themselves.

Once the angle $\theta$ is known, the height of the sensor $h_{m}$ above the sensor datum can be determined using:

$$
h_{m}=\left(\frac{1}{n} \sum_{i=1}^{n} d_{i}\right) \cos \boldsymbol{\theta}
$$

where the $d_{i}$ are the $n$ measurements made to the surface of the ground. In cases where the standing residue is sparse and many ground points are recorded, smoothing can be used to define a continuous datum, which is also indicative of pattern roughness such as furrows or tillage ridges.

Horizontal positioning of the laser sensor was implemented using a traversing rail moved by a stepper motor. Position information could be determined to within $0.02 \mathrm{~mm}$. The laser was mounted to the rail with $\theta$ equal to 45 degrees and $h_{m}$ approximately $1200 \mathrm{~mm}$ above the soil surface. Readings were taken every 0.3 millimeter along the length of the rail. The experimental target consisted of wheat straw stems inserted into a sheet of Styrofoam insulation in a variable density, row-oriented pattern. A thin layer of dry soil was spread on the surface to provide a natural background for the measurement. To determine the absolute reference value of the silhouette area index, the number of stems was hand counted, and a sampling of stem diameters and heights was measured. For the 46 stems measured, the average diameter was $3.4 \mathrm{~mm}$, the maximum diameter was $4.1 \mathrm{~mm}$ and the minimum diameter was $2.4 \mathrm{~mm}$. After placing the standing stems, of 56 measured, the average height was $244 \mathrm{~mm}$, th maximum height was $276 \mathrm{~mm}$, and the minimum height was $208 \mathrm{~mm}$. Scans were taken at three locations along the row pattern and two locations across the row pattern.

\section{Results}

The times required to obtain the scans presented were on the order of 20 seconds for each distance of $600 \mathrm{~mm}$. Distance readings showed the location of th s soil surface and the location of stems. At the position where the laser spot partially illuminated the stem and partially illuminated the soil surface, distance readings were greater than the distance to the soil surface. Experimentation with the laser on single stems and a metal calibration block showed that this pattern occurs consistently when the laser light spot is straddling an extreme distance transition.

In order to successfully extract a silhouette area index from a scan of this type, both the location of the soil surface and the area of standing residue above the soil surface must be determined. The following characteristics of the scan were used to identify the soil surface. First, the slope between points on the soil surface is less than the slope between points defining the start of standing residue. Second, the width of standing residue is small compared to the width of soil surface regions. Lastly, the relief of the soil surface is less than the relief of the standing residue. Therefore the algorithm for soil surface identification separates high and low slope regions, wide and narrow regions, and high and low relief regions. The algorithm was able to identify the soil surface in all the scans processed.

Once the soil surface has been identified, the silhouette area index can be calculated. Using the soil surface as a reference, the previously identified high relief regions are added to the width of standing residue $\left(w_{\text {above threshold }}\right)$, if they exceed some threshold $\left(h_{\text {threshold }}\right)$ above the soil surface. Making the assumption that the height of the standing residue is relatively uniform, the silhouette area of standing residue is simply the maximum height above the soil surface $\left(h_{\max }\right)$ times the width of standing residue in the scan. The area of the soil surface covered by the scan is found using the total traverse length of the scan $\left(w_{\text {scan }}\right)$ times the horizontal distance measured by the laser. This is expressed in the equation:

$$
s a i=\frac{h_{\max } * w_{\text {above threshold }}}{w_{\text {scan }} *\left(h_{\max }-h_{\text {threshold }}\right) * \tan \boldsymbol{\theta}}
$$

where $\theta$ is the angle of the laser beam as defined in Figure 1. Using these algorithms with a slope threshold of 30, a width threshold of $6 \mathrm{~mm}$, and a relief threshold of $30 \mathrm{~mm}$, the maximum stem height was found and silhouette area index was computed for each of the scans. The results are displayed in Table 1. 
Table 1. Comparison of counted and scanned silhouette area indexes and stem heights

\begin{tabular}{|l|l|l|l|l|l|}
\hline \multirow{2}{*}{ Scan Direction } & \multicolumn{3}{|c|}{ Silhouette Area Index } & \multicolumn{2}{c|}{ Stem Height } \\
\cline { 2 - 6 } & Counted & Scanned & Ratio & $\begin{array}{l}\text { Measured } \\
\text { Average }\end{array}$ & $\begin{array}{l}\text { Scanned } \\
\text { Maximum }\end{array}$ \\
\hline \multirow{3}{*}{ Across Row } & 0.38 & 0.23 & 0.61 & 244 & 235 \\
\cline { 2 - 6 } & 0.38 & 0.20 & 0.53 & 244 & 233 \\
\hline \multirow{3}{*}{ Along Row } & 0.61 & 0.30 & 0.50 & 245 & 250 \\
\cline { 2 - 7 } & 0.48 & 0.24 & 0.50 & 240 & 265 \\
\cline { 2 - 7 } & 0.21 & 0.12 & 0.57 & 240 & 232 \\
\hline
\end{tabular}

\section{Discussion}

The laser distance measurement anomaly that occurs on the edge of sharp distance transitions accentuates the distinction between standing residue and the soil surface, aiding the discrimination between the two. The additional impact of this effect is that part of the width of the stem is lost in the transition zone. This is likely part of the reason that the scanned silhouette area index was consistently less than half of the counted silhouette area index. Future tests with larger stem sizes or finer scan resolution are planned to quantify this factor.

Another factor that may contribute to the lower scanned silhouette area index is the hiding of stems that occurs in dense stands. At the densities scanned in this study, little if any hiding occurred, because the ratios for all scans along the row were the same regardless of density. In wind erosion research, most of the measurements of interest would be for standing residue densities less than the values measured here.

With the relatively uniform height of the placed stems, the scanned heights were relatively close to the measured average height. In stands with highly variable stem height, use of actual scanned height for each stem would reduce the scanned sai even further.

\section{Conclusions}

The laser distance method of measuring a silhouette area index for standing residue provided consistent results for the limited number of scans obtained. The laser method consistently underestimated the silhouette area index when compared to hand counts with height and diameter measurements. A constant ratio of scanned to counted silhouette area index across varying densities of standing residue indicates that all stems are being counted and consistent measurements are being obtained. A low ratio indicates that stem silhouette area is being underestimated. Sampling time is about 1 minute for a 2 meter scan.

\section{References}

Hagen, L. J. 1996. Crop residue effects on aerodynamic processes and wind erosion. Theor. and Appl. Climatol., 54:39-46.

Hagen, L. J. and D.V. Armbrust. 1994. Plant canopy effects on wind erosion saltation. Trans. ASAE 37(2): 461-465.

Musick, H. B., Trujillo, S. M., and Truman, C. R., 1996. Wind-tunnel modeling of the influence of vegetation structure on saltation threshold. Earth Surf. Proc. and Landforms 21:589-605. 